\title{
Morse Theory and the Topology of Configuration Space
}

\author{
W.D.McGlinn ${ }^{\dagger}$, L.O’Raifeartaigh ${ }^{\ddagger}$, \\ S.Sen ${ }^{\sharp}$, R.D.Sorkin* \\ $\dagger$ Physics Department, University of Notre Dame, Notre Dame, IN 46556, USA \\ $\ddagger$ Dublin Institute for Advanced Studies, 10 Burlington Road, Dublin 4, Ireland \\ \# School of Mathematics, Trinity College, Dublin 2, Ireland \\ *Physics Department, Syracuse University, Syracuse, NY 13244-1130, USA and \\ Departamento de Gravitación y Teoría de Campos Instituto de Ciencias Nucleares, \\ UNAM A. Postal 70-543 México, D. F. 04510, México
}

\begin{abstract}
The first and second homology groups, $H_{1}$ and $H_{2}$, are computed for configuration spaces of framed three-dimensional point-particles with annihilation included, when up to two particles and an antiparticle are present, the types of frames considered being $S^{2}$ and $S O(3)$. Whereas a recent calculation for two-dimensional particles used the Mayer-Vietoris sequence, in the present work Morse Theory is used. By constructing a potential-function none of whose critical indices is less than four, we find that (for coefficients in an arbitrary field $K$ ) the homology groups $H_{1}$ and $H_{2}$ reduce to those of the frame-space, $S^{2}$ or $S O(3)$ as the case may be. In the case of $S O(3)$-frames this result implies that $H_{1}$ (with coefficients in $\mathbf{Z}_{2}$ ) is generated by the cycle corresponding to a $2 \pi$-rotation of the frame. (This same cycle is homologous to the exchange loop: the spin-statistics correlation.) It also implies that $\mathrm{H}_{2}$ is trivial, which means that there does not exist a topologically nontrivial Wess-Zumino term for $S O(3)$-frames (in contrast to the two-dimensional case, where $S O(2)$-frames do possess such a term). In the case of $S^{2}$-frames (with coefficients in $\mathbb{R}$ ), we conclude $H_{2}=\mathbb{R}$, the generator being in effect the frame space itself. This implies that for $S^{2}$-frames there does exist a Wess-Zumino term, as indeed is needed for the possibility of half-integer spin and the corresponding fermi statistics. Taken together, these results for $H_{1}$ and $H_{2}$ imply that our configuration space "admits spin-1/2" for either choice of frame, meaning that the spin-statistics theorem
\end{abstract}


previously proved for this space is not vacuous. 


\section{Introduction}

Results concerning spin-statistics correlations for extended objects such as solitons, monopoles and vortices have shown ${ }^{1}$ that the axioms of local relativistic quantum field theory are by no means necessary to guarantee a spin-statistics theorem ${ }^{2}$. The question of what general ${ }^{2}$ assumptions are needed for a spin-statistics theorem has led to the investigation ${ }^{3,4}$ of the general topological properties of systems of particles and extended objects, with the result that the physical consequences of these topological properties are now better understood. The importance of pair-creation and annihilation is clear. ${ }^{4,5}$

The topology of configuration space plays a important role in quantum theory. The quantum mechanical Hilbert space corresponding to a classical configuration space $C$ is in general best viewed as being the space of sections of a vector bundle over $C$. For a variety of physically interesting configuration spaces $C$, these vector bundles incorporate the spin-type and the statistics, as well as other topological properties of the quantum theory in question. Furthermore many of the relevant topological properties can be described by the homotopy and homology elements of the classical configuration spaces which are associated with these vector bundles.

For example, the set of $\mathrm{U}(1)$ (and hence line) bundles over $C$ is classified by $H^{2}(C ; Z)$, which is isomorphic to $H_{2}(C ; Z)^{*} \oplus$ Tor $H_{1}(C ; Z)$, where $H_{2}(C ; Z)^{*}$ can be thought of as the non-torsion part of $H_{2}(C ; Z)$ and $\operatorname{Tor}_{1}(C ; Z)$ is the torsion subgroup of $H_{1}(C ; Z){ }^{6}$ (The torsion subgroup of an abelian group is its maximal finite subgroup.) Also, in the case of (locally) flat bundles, the spin-statistics correlation follows from the statement that the exchange of two identical particles and a $2 \pi$ rotation of one of the particles both correspond to the same nontrivial element of the fundamental group $\pi_{1}(C)$. For more general bundles it rests (in three dimensions) on a homotopy, not between loops but between a certain pair of mappings of $\mathbb{R} P^{2}$ into $C{ }^{7}$ Similarly, the condition for the existence of a nontrivial Wess-Zumino term is that the second homology group $H_{2}(C ; Z)$ contain a $\mathrm{Z}$ subgroup, or equivalently that $H_{2}(C ; \mathbb{R})$ be non-zero. 
A system that has been investigated ${ }^{8,9}$ using this type of approach is that of identical particles and anti-particles on $\mathbb{R}^{d}$, each carrying a 'frame' $\mathrm{F}$, the frame having been introduced in order to describe intrinsic spin. ${ }^{8,9}$ In this case, the classical configuration space $C$ is (as a set) of the form $C=\bigsqcup Q_{m, n}$ where the $Q_{m, n}$ are spaces containing $m$ particles and $n$ anti-particles, all with distinct locations (but see Ref. 10), and the disjoint union $\sqcup$ runs over all possibilities for $m$ and $n$. The topology of each subspace $Q_{m, n}$ (or just $Q_{n}$ if the particle is its own antiparticle) is the topology of an appropriate frame bundle modified by the fact that the particles are assumed to be indistinguishable. The basic problem (solved in Ref. 9) is to construct a (Hausdorff) topology for the full space $C$ such that pair creation and annihilation can proceed continuously. The problems of finding the precise topological properties that are introduced by the construction of $C$ and of analyzing these properties remains.

In Ref. 11 we considered a limited version of this problem wherein the individual units are point particles which move in two-dimensional Euclidean space $\mathbb{R}^{2}$ and carry 'frames' which embody the notion of spin. By 'carrying frames' is meant that a single particle (or anti-particle) is represented by a bundle over $\mathbb{R}^{2}$. Three possible fibers, namely $S O(2), S^{2}$, and $S O(3)$, were considered. The restriction to point particles and to two dimensions was for simplicity and because of the present interest in two-dimensional systems, particularly in the theory of "anyons". In this paper we expand the earlier study to include particles which move in three-dimensional Euclidean space $\mathbb{R}^{3}$ and carry frames $S^{2}$ or $S O(3)$.

The solution to the problem of finding an appropriate topology for the configuration space is reviewed in Section 2. The essential idea is to introduce open neighborhoods of the vacuum (and corresponding neighborhoods of the non-vacuum configurations) that allow a particle and an antiparticle to annihilate provided their positions and frames are suitably aligned. (For an isolated particle-antiparticle pair, their frames must be "mirror images" of each other; if a further particle is nearby in space, we require in addition that the two particles be on opposite sides of the 
antiparticle with both pairs of frames matched, a situation which we call 'syzygy' in analogy with planetary alignments). The complete topology is then obtained from these neighborhoods. There remains the problem of determining in detail the properties of the resulting topological space, including its homology and homotopy groups, especially insofar as they help answer the question of how many inequivalent vector bundles the space admits.

In the present work, we will again concentrate on the homology groups $H_{1}$ and $H_{2}$, these providing complete information if we restrict ourselves to line bundles. In Ref. 11 we determined these groups for particles moving in $\mathbb{R}^{2}$ by using the Mayer-Vietoris exact sequence. This process was carried out for the first and second homology groups in the case of the subspaces $X_{1,1}=\bar{Q}_{1,1}=Q_{1,1} \cup Q_{0,0}$ and $X_{2,1}=$ $\bar{Q}_{2,1}=Q_{2,1} \cup Q_{1,0}$. In this paper we solve for the first and second homology groups for particles moving in three-dimensional Euclidean space $\mathbb{R}^{3}$, carrying frames $S^{2}$ or $S O(3)$. A result of particular importance we find is that for $S O(3)$-frames, $H_{1}\left(X_{2,1} ; Z_{2}\right)=Z_{2}$, the non-zero element being the exchange, or equivalently the $2 \pi$ rotation of a single frame. (That these two 1-cycles are homologous expresses the spin-statistics correlation.) This implies that the particles will be spinorial fermions for an appropriate choice of line bundle, and thereby demonstrates the nontriviality of our framework (at least up to $\left.X_{2,1}\right)$. Similarly we find that $H_{2}\left(X_{2,1} ; \mathbb{R}\right)=\mathbb{R}$ for $S^{2}$-frames, showing non-triviality in this, somewhat more general case as well [see Ref. 7]. In addition we find that $H_{2}$ is trivial for $S O(3)$-frames.

The layout of the paper is as follows. In Sec. 2 we describe the topology of the spaces to be considered. In Secs. 3 and 4, we discuss how Morse Theory can be used to obtain information about homology groups. A Morse potential applicable to our spaces is described in Secs. 5 and 6. In Sec. 7 its critical points are found, and in Sec. 8 the critical indexes of these points are determined and the implications for $H_{1}$ and $H_{2}$ are drawn. Sec. 9 contains a discussion of the directions that generalizations of the results of this paper may take. 
2 TOPOLOGY OF THE SPACE OF FRAMED POINT-PARTICLES AND ANTIPARTICLES5

\section{Topology of the Space of Framed Point- Particles and Antiparticles}

The topology of the space of framed point-particles and antiparticles is described in detail in Ref. 9. For completeness, we give a brief description of this space, including the 'reflection' and 'syzygy' conditions for annihilation. Let $X=\left(x, F^{(x)}\right)$, [respectively $\bar{X}=\left(\bar{x}, \bar{F}^{(\bar{x})}\right)$ ] denote the position and 'frame-orientation' of a particle [resp. antiparticle]. By $F^{(x)}$ we mean a generic 'frame' (in $S^{2}, S O(3)$ or $S O(2)$ as the case may be) attached to the particle located at position $x$. Then

$$
\begin{aligned}
Q_{m, n}= & \left\{\left[X^{1}, X^{2}, \cdots, X^{m} ; \bar{X}^{1}, \bar{X}^{2}, \cdots, \bar{X}^{n}\right] \mid x^{i}, \bar{x}^{j} \in \mathbb{R}^{d} ;\right. \\
& \left.x^{i} \neq x^{j}, \bar{x}^{i} \neq \bar{x}^{j} \text { if } i \neq j ; x^{i} \neq \bar{x}^{j}\right\}
\end{aligned}
$$

is the sector or "stratum" of our configuration space describing $m$ particles and $n$ antiparticles. Here, the bracket notation indicates that the order of the $X^{i}$ is without significance (and similarly for the $\bar{X}^{j}$ ). We also introduce the vacuum ("VAC") by setting

$$
Q_{0,0}=\{V A C\}
$$

Next the concept " $\epsilon$-close" is defined as follows: (i) Particles $\mathrm{X}$ and $\mathrm{Y}$ are " $\epsilon$ close" iff $|x-y|<\epsilon$ and $d\left(F^{(x)}, F^{(y)}\right)<\epsilon / L$ (where $\mathrm{L}$ is some fixed length), and similarly for antiparticles $\bar{X}$ and $\bar{Y}$; (ii) The particle $\mathrm{X}$ and antiparticle $\bar{X}$ are " $\epsilon$ close" iff

$$
|x-\bar{y}|<\epsilon \quad \text { and } \quad d\left(F^{(x)}, R(x-\bar{x}) \bar{F}^{(\bar{y})}\right)<|x-\bar{y}| / L .
$$

Here $|x-\bar{y}|$ is the Euclidean distance between points $x$ and $\bar{y}, d\left(F^{(x)}, F^{(y)}\right)$ is the geodesic distance between $F^{(x)}$ and $F^{(y)}$ in the space of frames, and $R(x-$ $\bar{y}) \bar{F}^{(\bar{y})}$ is the frame which results when the anti-frame $\bar{F}^{(\bar{y})}$ is reflected in the plane perpendicular to the vector $x-\bar{y}$. This concept of " $\epsilon$-close" is used to define an $\epsilon$-neighborhood in $Q_{m, n}$ of a point in $Q_{m, n}$ in the obvious way.

We further need to define when a point

$$
\mathbf{Y}=\left[Y^{1}, \cdots, Y^{m+p} ; \bar{Y}^{1}, \cdots, \bar{Y}^{n+p}\right] \in Q_{m+p, n+p} \quad, p \geq 0
$$


is in an $\epsilon$-neighborhood of a point

$$
\mathbf{X}=\left[X^{1}, \cdots, X^{m,} ; \bar{X}^{1}, \cdots \bar{X}^{n}\right] \in Q_{m, n}
$$

that is when $p$ particle-antiparticle pairs are 'close to annihilation'. To this end we define a viable labeling of $\mathbf{Y}$ with respect to $\mathbf{X}$ as one that satisfies the following: (i) $Y^{i}$ is $\epsilon$-close to $X^{i}$ for $\mathrm{i}=1, \ldots, m$; (ii) $\bar{Y}^{i}$ is $\epsilon$-close to $\bar{X}^{i}$ for $\mathrm{i}=1, \ldots, n$; (iii) $Y^{i+m}$ is $\epsilon$-close to $\bar{Y}^{i+n}$ for $\mathrm{i}=1, . . p$. Also, we say that a triplet $X, Y, \bar{Z}$ is in syzygy if (and only if)

$$
\frac{|x-\bar{z}|+|y-\bar{z}|}{L}>\left\{\begin{array}{l}
|\widehat{x-\bar{z}}+\widehat{y-\bar{z}}| \\
d\left(F^{(x)}, R(x-\bar{z}) \bar{F}^{(\bar{z})}\right) \\
d\left(F^{(y)}, R(y-\bar{z}) \bar{F}^{(\bar{z})}\right)
\end{array}\right.
$$

A similar definition of syzygy applies to the triplet $\bar{X}, \bar{Y}, Z$. Here $\widehat{x-\bar{z}}$ indicates the unit vector in the direction $x-\bar{z}$. Finally we say that $\mathbf{Y} \in N_{\epsilon}[\mathbf{X}]$, that is, $\mathbf{Y}$ is an element of the $\epsilon$-neighborhood of $\mathbf{X}$, if there exists a viable labeling of $\mathbf{Y}$ with respect to $\mathbf{X}$, and for all such viable labelings, all suitable triplets are in syzygy. By suitable triplets we mean that at least one member of the triplet comes from the set $\left\{Y^{m+1}, \cdots, Y^{m+p} ; \bar{Y}^{n+1}, \cdots, \bar{Y}^{n+p}\right\}$, that is one 'new' particle or one 'new' antiparticle must be a member of the triplet.

In summary, the condition that a particle-antiparticle pair be "close to annihilation" is first of all that they be spatially close to each other and that their frames nearly satisfy the reflection condition. In addition, if another particle is nearby in space, the three must be in syzygy. This means that the two particles are on opposite sides of the antiparticle and both particles nearly satisfy the reflection condition with the antiparticle.

\section{Morse Theory}

Morse Theory ${ }^{12}$ relates the homology groups of a manifold $M$ to the so-called critical indices of a suitable smooth function $V$ on $M$. Critical points of $V$ are points $p$ at 
which the gradient of $V$ vanishes: $\left.d V\right|_{p}=0$. The number of negative eigensigns of the Hessian $\left.d^{2} V\right|_{p}$ at such a $p$ is referred to as the index of $p$ (relative to $V$ ), and $V$ is said to be nondegenerate if (nullity of $p$ ) $:=\operatorname{dim} M-\operatorname{rank} d^{2} V_{p}=0$.

Let us assume that each of the sub-spaces $M_{a}=\{s \in M \mid V(s) \leq a\}$ is compact. If $p$ is the only critical point of $V$ in the range $V(p)-\epsilon<V(p)<V(p)+\epsilon$ and $p$ is nondegenerate of index $\lambda$, Morse theory tells us that $M_{V(p)+\epsilon} \approx M_{V(p)-\epsilon} \cup e_{\lambda}$ where $e_{\lambda}$ is a $\lambda$ - cell and ' $\approx$ ' denotes homotopy equivalence. (Considering a Morse function as a potential, it effectively induces a retraction of $M_{V(p)+\epsilon}$ to $M_{V(p)-\epsilon} \cup e_{\lambda}$.) Because attaching $e_{\lambda}$ must create either a new cycle or a new boundary, this in turn implies that either

$$
\begin{aligned}
\text { 1) } \operatorname{dim}_{\lambda}\left(M_{V(p)+\epsilon} ; K\right) & =\operatorname{dim}_{\lambda}\left(M_{V(p)-\epsilon} ; K\right)+1 \text { or } \\
\text { 2) } \operatorname{dim} H_{\lambda-1}\left(M_{V(p)+\epsilon} ; K\right) & =\operatorname{dim} H_{\lambda-1}\left(M_{V(p)-\epsilon} ; K\right)-1
\end{aligned}
$$

Here $K$ is an arbitrary field and $\operatorname{dim}_{\lambda}(M ; K)$ is the dimension of the $\lambda^{\text {th }}$ homology group of $M$ with coefficients in $K$ (i.e. its dimension as a vector space over $K$ ). To discover whether alternative 1 or 2 obtains requires a global analysis.

If one exhibits a smooth function $V$ on a closed manifold $M$ for which all the critical points in $M \backslash M_{0}$ are nondegenerate (hence finite in number), then $M$ can be constructed by the successive attachment of thickened $\lambda_{i}$-cells to $M_{0}$, where $\lambda_{i}$ is the index of the critical point $p_{i}$. In particular, according to (4), dim $H_{\lambda}$ can change in going from $M_{0}$ to $M$ only if $\lambda=\lambda_{i}$ or $\lambda=\lambda_{i}-1$ for some $i$. From this it follows that, if $\lambda_{i} \geq 4$ for all $p_{i}$, then $\operatorname{dim} H_{j}(M ; K)=\operatorname{dim} H_{j}\left(M_{0} ; K\right), j=1,2$. It is this result that we will use.

Actually we will need results which generalize the above. First, for 'natural' Morse functions defined on our configuration spaces the critical points $p_{i}$ will comprise critical submanifolds $N_{i}$ which reflect an overall rotation invariance, as well as certain other symmetries. We can still apply Morse theory if the Hessian $d^{2} V_{p_{i}}$ is nondegenerate in the directions normal to $N_{i}$, and one still defines the index of $N_{i}$ as the number of negative eigensigns of that Hessian. Second, the spaces we will consider are not globally manifolds nor are they compact. The generalizations 
needed will be described in the next section.

\section{Sufficient Conditions for Applying Morse Theory}

We would like to have information about the homology groups of the space $X_{2,1}:=$ $\bar{Q}_{2,1}=Q_{1,0} \cup Q_{2,1}$, which is not a manifold at points of its lower-dimensional "stratum" $Q_{1,0}$. Nevertheless, we can still use Morse theory to get information on $\operatorname{dim}_{i}\left(X_{2,1} ; K\right)-\operatorname{dim}_{i}\left(Q_{1,0} ; K\right)$. We have shown elsewhere ${ }^{11}$ that thickening $Q_{1,0}$ into $Q_{2,1}$ to obtain the $\epsilon$-neighborhood $\widetilde{Q}_{1,0}=N_{\epsilon}\left[Q_{1,0}\right]$ does not alter the homology of $Q_{1,0}$; in fact $\widetilde{Q}_{1,0}$ retracts onto $Q_{1,0}$ and is therefore homotopy equivalent to it. Outside of $\widetilde{Q}_{1,0}$ we do have a manifold (a subset of the top-dimensional "stratum" $\left.Q_{2,1}\right)$, and can therefore hope to use Morse theory to reduce the study of $X_{2,1}$ to that of $\widetilde{Q}_{1,0}$, and thereby to $Q_{1,0}$ itself.

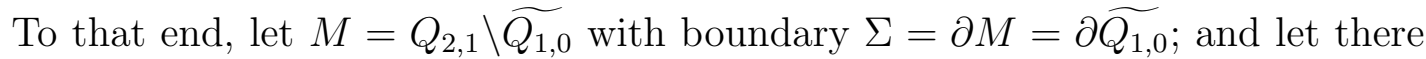
be given on $M$ a Riemannian metric $g_{a b}$ and a smooth positive potential-function $V$. As earlier, we write $M_{a}=V^{-1}([0, a])$. A sufficient set of conditions to apply Morse theory to $X_{2,1}$ in the indicated manner is the following.

1. $\widetilde{Q}_{1,0}$ retracts onto $Q_{1,0}$ (or onto any subset thereof onto which $Q_{1,0}$ itself retracts);

2. $\Sigma=\partial M$ is a piece-wise smooth submanifold of $Q_{2,1}$;

3. The gradient flow $\xi^{a}=-g^{a b} \partial_{b} V$ is inward everywhere on $\Sigma$ (by inward we mean into $\widetilde{Q}_{1,0}$, which is outward from the point of view of $M$ );

4. The critical submanifolds of $V$ are compact (and nondegenerate), and there are a finite number of them in each $M_{a}$;

5. The vector flow $\xi^{a}$ leads each $x \in M$ either to a point of $\Sigma$ or to a critical submanifold of $V$; 
6. The potential $V$ increases without bound along any trajectory of the inverse vector flow $-\xi^{a}$ (unless the trajectory hits a critical point).

The argument that the above conditions suffice follows the same steps as the corresponding chain of reasoning from ordinary Morse theory, the main differences being first, that $M$ is non-compact, and second, that the starting surface $\Sigma$ from which $M$ gets built up is not a level surface of $V$. (With our Morse function, there will be equipotential surfaces of large $V$ intersecting $\Sigma$, essentially because of the influence of the term $V_{\epsilon}$, which we will introduce in the next section for the sake of satisfying conditions 5 and 6 above.)

To cope with the circumstance that $V$ is not constant on $\Sigma$, we introduced a slightly modified notation, letting

$$
\begin{aligned}
M_{t} & =\Sigma \cup V^{-1}([0, t]) \\
& =\{x \in M \mid x \in \Sigma \vee V(x) \leq t\}
\end{aligned}
$$

and

$$
\Sigma_{t}=V^{-1}(t) \cup\{x \in \Sigma \mid V(x)>t\}
$$

Thus, $M_{t}$ comprises the $V<t$ subset of $M$ together with that portion of $\Sigma$ through which the $V<t$ subset does not yet "protrude"; and $\Sigma_{t}$ is what might be called "the future boundary of $M_{t}$ " (see Figure 1). Note that since $V>0$ everywhere, $\Sigma_{0}$ $=\Sigma=\partial M\left(=\partial \widetilde{Q}_{1,0}\right)$. (Notice also that $M_{t}$ is not strictly a manifold, but would be if we thickened $\Sigma$ slightly "back into" $\widetilde{Q}_{1,0}$.) We will denote the critical values of $V$, taken in increasing order, by $v_{1}, v_{2}, \ldots$, each of which we assume to correspond to a single connected critical submanifold $N_{i}$ of $V$.

As usual, we consider the sequence of subspaces $M_{t}$ with $t$ increasing from 0 to $\infty$, and we argue first that nothing happens between critical values, and second that in passing a critical value $v_{i}$ we merely in effect attach a cell of dimension $\lambda_{i}$ to $M, \lambda_{i}$ being the index of the $i^{\text {th }}$ critical point, as in the discussion of the

\footnotetext{
${ }^{1}$ Another approach here might be to use the flow associated with $\xi$ for a "Morse-Smale analysis". This could offer a different way around the difficulty that $\Sigma$ is not a level surface of $V$.
} 
previous section. Actually, since we will be dealing with critical submanifolds this last statement must be modified, but the only difference is that now, instead of the homology of $M$ being altered in dimension $\lambda$ or $\lambda-1$ according to equation (4), it can be altered in higher dimensions as well. (A more precise statement is that, in passing a critical submanifold $N$ of index $\lambda$, it is as if $b_{k}$ cells had been added in dimension $\lambda+k, k=0,1,2 \ldots$, where $b_{k}$ is the $k^{\text {th }}$ betti number of $N$ with coefficients in a certain orientation-bundle over $N$ (see ref. 12). For our purposes this makes no difference, and in the following discussion, we will speak as if all the critical manifolds were simply (non-degenerate) critical points.

To begin with, let us argue that $M_{t} \approx \Sigma_{0}$ for all $t<v_{1}(\approx$ denoting homotopy equivalence). For $t \leq 0, M_{t}=\Sigma=\Sigma_{0}$ and our claim is trivially true. So consider any $t>0$ but still less than $v_{1}$. In order to retract $M_{t}$ back to $\Sigma$, let us introduce on $M_{t}$ the renormalized Morse flow,

$$
\hat{\xi}^{a}(x)=\frac{-g^{a b}(x) \partial_{b} V(x)}{g^{c d}(x) \partial_{c} V(x) \partial_{d} V(x)} \frac{V(b(x))-V(a(x))}{t},
$$

where, for each $x \in M_{t}, a(x)$ is the unique point of $\Sigma_{0}$ to which $x$ flows via the gradient flow, and $b(x)$ is the unique point of $\Sigma_{t}$ to which it flows via the reversed gradient flow. (In other words, $a$ and $b$ are the intersections of $\Sigma$ and $\Sigma_{t}$ with the gradient flow-line through $x$.) Since there are no critical points within $M_{t}, \hat{\xi}^{a}$ is nonzero and smooth, except possibly on $\Sigma \cap\{V=t\}$, where it vanishes. Moreover, $\hat{\xi}^{a}$ has been normalized so that $\hat{\xi}^{a}(x) \partial_{a} V(x)=-(V(b(x))-V(a(x))) / t$, which means that the parameter difference along $\hat{\xi}^{a}$ between $\Sigma_{t}$ and $\Sigma_{0}=\Sigma$ has the constant value $t$. Thus the flow induced by $\hat{\xi}^{a}$ effects a retraction of $M_{t}$ back onto $\Sigma_{0}$, whence these two spaces are homotopic as claimed. (By retraction, we always mean deformation retract.)

To handle the similarly uneventful transition from $t=v_{i}+\epsilon$ to $t=v_{i+1}-\epsilon$ we can argue in the same manner that $M^{\prime}:=M_{v_{i+1}-\epsilon} \backslash \stackrel{\circ}{M_{v_{i}+\epsilon}}=M_{v_{i+1}-\epsilon} \backslash\left\{V<v_{i}+\epsilon\right\}$ retracts onto $\Sigma^{\prime}:=\Sigma_{v_{i}+\epsilon}$ and therefore that $M_{v_{i+1}-\epsilon}$ retracts onto $M_{v_{i}+\epsilon}$ as desired. This proceeds as before with $M^{\prime}$ playing the role of $M$ and $\Sigma^{\prime}$ the role of $\Sigma$, it being clear in particular that $\xi$ has no zeroes in $M^{\prime}$ and that each $x \in M^{\prime}$ flows to some 
point of $\Sigma^{\prime}$. Similarly, nothing of note occurs beyond the final critical value $v_{N}$ (if there is one), since there, a homotopy based on the Morse flow itself, i.e. on the vectorfield $\hat{\xi}^{a}=-g^{a b} \partial_{b} V / g^{c d} \partial_{c} V \partial_{d} V$, can be used to retract $M$ back to $M_{v_{N}+\epsilon}$.

Finally, we must analyze what happens in passing a critical value, say $v_{i}$ with associated critical point $x_{i}$. The key observation is that, since everything really happens in a neighborhood of $x_{i}$ (or more generally in a neighborhood of the compact critical submanifold $N_{i}$ ), the effect on $M_{t}$ is just as it is in the standard situation discussed in the last section. More precisely, it follows from the analysis which applies in that situation (see e.g. ref. 12) that there exist a standard neighborhood $\mathcal{U}$ of $x_{i}$, and decompositions

$$
M^{+} \backslash \stackrel{\circ}{M^{-}}=\mathcal{U} \sqcup \mathcal{U}^{\prime} \quad\left(M^{ \pm}:=M_{v_{i} \pm \epsilon}\right)
$$

and

$$
\Sigma^{-}=S \sqcup S^{\prime} \quad\left(\Sigma^{ \pm}:=\Sigma_{v_{i} \pm \epsilon}\right)
$$

such that

(i) the flow $\hat{\xi}$ retracts $\mathcal{U}^{\prime}$ onto $S^{\prime}$, just as above;

(ii) there exists the usual Morse-theoretic retraction of $\mathcal{U}$ onto $S \cup e_{\lambda}$ (i.e. a space made by attaching a $\lambda$-cell to $S$ ); and

(iii) the two retractions agree on $\overline{\mathcal{U}} \cap \overline{\mathcal{U}^{\prime}}$.

Then the combined retractions in $\mathcal{U}$ and $\mathcal{U}^{\prime}$ homotope $M^{+} \backslash \stackrel{\circ}{M^{-}}$to $\Sigma^{-} \cup e_{\lambda}$, hence they also retract $M^{+}$onto $M^{-} \cup e_{\lambda}$, as required. (See figure 2.)

Applying this analysis to the case at hand, we conclude that when the conditions enumerated above are fulfilled, $\overline{Q_{2,1}}$ is in effect built up from $Q_{1,0}$ by adding cells of dimensions not less than the smallest index $\lambda_{\min }$ of any critical submanifold of $V$. It follows that $H_{i}\left(X_{2,1} ; K\right)=H_{i}\left(Q_{1,0} ; K\right)$ for all $i \leq \lambda_{\text {min }}-2$.

\section{A Morse Function for $Q_{2,1}$}

Consider the following potential. 


$$
V=\left(\rho_{1}+\frac{x_{1}{ }^{2}}{10 \rho_{1}}\right)\left(\rho_{2}+\frac{x_{2}{ }^{2}}{10 \rho_{2}}\right)\left(\frac{1}{16}+\frac{x+x_{1}+x_{2}}{\left(\rho_{1}{ }^{2}+\rho_{2}{ }^{2}+2 \rho_{1} \rho_{2}(1-x)\right)^{\frac{3}{4}}}\right)
$$

Here (with respect to an arbitrary labeling of the particles) $\rho_{i}=\left|x_{i}-\bar{y}\right|$ is the distance between particle $i$ and the antiparticle, $x_{i}=1-\cos \left(\theta_{i}\right)$ with $\theta_{i}$ the angular separation $d\left(F^{\left(x_{i}\right)}, R\left(x_{i}-\bar{y}\right) \bar{F}^{(\bar{y})}\right)$, and $x=1-\cos (\theta)$ with $\theta$ the angle between $-\overrightarrow{\rho_{1}}$ and $\overrightarrow{\rho_{2}}$ so that $\rho^{2}=\rho_{1}^{2}+\rho_{2}^{2}+2 \rho_{1} \rho_{2}(1-x)$ is the distance squared between the two particles; and we have taken $L=1$.

The potential is the product of three factors. The first two factors drive the two particles to satisfy the reflection requirement with the antiparticle and to move toward annihilation with it, thus tending to produce a flow of $Q_{2,1}$ toward $Q_{1,0}$, as we desire. Nevertheless there is no obvious guarantee that points in $Q_{2,1}$ are not in some cases driven toward two types of "internal boundaries": 1) where one or both of the particles are near to the antiparticle but not close to annihilation or 2) where the two particles are close to each other but not close to the antiparticle. Either occurrence would violate condition 5 of the previous section. In fact, however, the 'internal boundary' of type 2 is not approached by the flow induced by $V$ because the $1 / \rho^{3 / 2}$ in the second term of the third factor drives the particles apart unless they are in syzygy. Despite this, $V$ can still become small if at at least one of the first two factors becomes small, but in that case at least one particle would have to draw near to the antiparticle, and we would be near a boundary of type 1 rather than type two.

In order to insure that an internal boundary of type 1 is also not approached we will add a 'small' term $V_{\epsilon}$ to the potential:

$$
V \rightarrow V+V_{\epsilon}, \quad V_{\epsilon}=\frac{\epsilon^{n}}{\rho_{1} \rho_{2}}
$$

where $n$ is some positive integer, say 6 , and $\epsilon$ is the $\epsilon$ of $N_{\epsilon}$. This term will drive the particles away from the antiparticle when they get very close to it (the coupling $\epsilon^{n}$ being very small), but on the other hand we will take $n$ large enough to avoid 
any violation of condition 3 of the previous section. The addition of $V_{\epsilon}$ also ensures that condition 6 is fulfilled in the neighborhood of the "internal boundary".

The following analysis assumes that the trivial translational degree of freedom has been removed by bringing the anti-particle to the origin via a preliminary retraction mapping. This will allow all the critical submanifolds to be compact.

We are now almost ready to apply Morse Theory, but first we must establish that our potential and metric lead to a flow which is inward everywhere along the boundary $\Sigma$.

\section{The Flow at the Boundary $\Sigma$}

In order to verify condition 3 , we need to characterize $\Sigma$, the boundary of $\widetilde{Q}_{1,0}$. In fact we use a slightly modified definition of $\widetilde{Q_{1,0}}$, as given by the following conditions (recall, we have set $L=1$ ):

1. $x_{1}<\rho_{1}<\epsilon$,

2. $x_{2}<\rho_{2}, x<\rho_{2}$.

More precisely the condition is that there exist a labeling for which 1 and 2 hold; and we will always use such a labeling. Moreover we will always choose it so that $\rho_{1} \leq \rho_{2}$, as is clearly possible since reversal of 1 and 2 will not invalidate the conditions when $\rho_{2} \leq \rho_{1}$. [In changing $\widetilde{Q}_{1,0}$ in this way we have in effect introduced a modified conception of neighborhood (modifying the condition for syzygy) which could be used to define a modified topology for $X_{2,1}=\bar{Q}_{2,1}$. The new definition would be very similar to the old one for small angles but easier to handle algebraically. Unless we actually alter the topology in this way, we do not guarantee that our new $\widetilde{Q_{1,0}}$ will be a true neighborhood of $Q_{1,0}$, but that doesn't matter: all we really need is that condition 1 of Sec. 4 still obtain for our new $\widetilde{Q}_{1,0}$, and it does.] The boundary $\Sigma$ is obtained by replacing any of the four inequalities comprising conditions 1 and 2 by an equality, for example $x_{1}=\rho_{1}$. 
There is considerable freedom in choosing the metric $g_{a b}$ which enters into the Morse flow, and in particular influences the direction of the flow across the boundary. We choose

$$
d s^{2}=\frac{\rho_{1}^{2}}{a\left(x_{1}\right)} d x_{1}^{2}+\frac{\rho_{2}^{2}}{a\left(x_{2}\right)} d x_{2}^{2}+\frac{\left(\rho_{1} \rho_{2}\right)^{2}}{a(x)} d x^{2}+d \rho_{1}^{2}+d \rho_{2}^{2},
$$

where $a(x)$ is chosen to vanish linearly at $x=0,2$, and to rise rapidly to a value of, say, 20 away from $x=0,2$. More specifically, we assume that $a(x) \sim x$ [resp. $2-x$ ] for $x \rightarrow 0$ [resp. 2]. Now (7) is not actually a metric on $Q_{2,1}$, but only on the 5 -dimensional quotient space of the parameters $\theta$ (or $x$ ) and $\rho$ (call this space $P$ ). Nonetheless, one can always choose a metric on $Q_{2,1}$ such that the flow computed on $P$ is compatible with that on $Q_{2,1}$. Since each point of $P$ corresponds to a compact submanifold of $Q_{2,1}$, this is all we will need. (The condition for compatibility is that the inverse metric $G^{A B}$ on $Q_{2,1}$ go over to the inverse of the metric (7) on $P$ under the natural projection of $Q_{2,1}$ onto $P$; in symbols $g^{a b}(y)=G^{A B}(x) \partial y^{a} / \partial x^{A} \partial y^{b} / \partial x^{B}$, where $y \in P$ and $x \in Q_{2,1}$.) By having the denominators of the angle terms in (7) vanish linearly at $x_{i}=0,2$ we ensure that $G^{A B}$ is nonsingular at $\theta_{i}=0, \pi$.

We now compute $\partial \vec{V} \cdot \vec{n}$ where $\vec{n}$ is a conveniently chosen outwardly-oriented (co-)vector normal to the boundary $\Sigma$ of $\widetilde{Q}_{1,0}$. (In expressing vectors as $n$-tuples, we take the coordinates in the order $\left(x_{1}, x_{2}, x, \rho_{1}, \rho_{2}\right)$.)

1. For that part of the boundary defined by $x_{1}=\rho_{1}<\epsilon$, we have, with $\vec{n}=$

$$
\begin{aligned}
(1,0,0,-1,0)=\partial x_{1}- & \partial \rho_{1}, \\
\frac{\partial \vec{V} \cdot \vec{n}}{\rho_{2}+\frac{x_{2}^{2}}{10 \rho_{2}}}= & \frac{11 a}{10 \rho_{1}^{2}}\left(\frac{\rho_{1}}{\left(\rho_{1}^{2}+\rho_{2}{ }^{2}+2 \rho_{1} \rho_{2}(1-x)\right)^{\frac{3}{4}}}\right) \\
& +\frac{a}{5 \rho_{1}^{2}}\left(\frac{1}{16}+\frac{\rho_{1}+x+x_{2}}{\left(\rho_{1}^{2}+\rho_{2}^{2}+2 \rho_{1} \rho_{2}(1-x)\right)^{\frac{3}{4}}}\right) \\
& +\left(\frac{33 \rho_{1}\left(2 \rho_{1}+2 \rho_{2}(1-x)\right)\left(\rho_{1}+x+x_{2}\right)}{40\left(\rho_{1}^{2}+\rho_{2}^{2}+2 \rho_{1} \rho_{2}(1-x)\right)^{\frac{7}{4}}}\right) \\
& -\frac{9}{10}\left(\frac{1}{16}+\frac{\rho_{1}+x+x_{2}}{\left(\rho_{1}^{2}+\rho_{2}^{2}+2 \rho_{1} \rho_{2}(1-x)\right)^{\frac{3}{4}}}\right)
\end{aligned}
$$


All terms are positive except the last. Consider the sum of the second (positive) term and the fourth (negative) term, that is

$$
\left(\frac{a\left(\rho_{1}\right)}{5 \rho_{1}^{2}}-\frac{9}{10}\right)\left(\frac{1}{16}+\frac{\rho_{1}+x+x_{2}}{\left(\rho_{1}^{2}+\rho_{2}^{2}+2 \rho_{1} \rho_{2}(1-x)\right)^{\frac{3}{4}}}\right)
$$

Since $\rho_{1}<\epsilon$, this is clearly positive (assuming $\epsilon \ll 1$ ) because $a \sim \rho_{1}$ for small $\rho_{1}$. Thus the flow is inward if we ignore the effect of $V_{\epsilon}$. However, since $V_{\epsilon}$ has no angle dependence $\partial V_{\epsilon} \cdot \vec{n}>0$ is also positive, which only makes things better.

2. For that part of the boundary defined by $x_{2}=\rho_{2}$, with $\vec{n}=(0,1,0,0,-1)=$ $\partial x_{2}-\partial \rho_{2}$, we obtain the same expression as in the previous case with 1 and 2 interchanged. If $\rho_{2}<\epsilon$, we are thus reduced to case 1 just treated, so we may as well assume that $\rho_{2} \geq \epsilon$. Then for $\rho_{2}$ not near the value 2 (where $a=0$ ), it is easy to see that again the sum of the second and last terms of equation 8 is positive, since its first factor, $a\left(\rho_{2}\right) / 5 \rho_{2}^{2}-9 / 10$, exceeds $1 / 10$ if $a\left(\rho_{2}\right)=20$. For $x_{2}=\rho_{2}$ very close to 2 , this factor does become negative, but in that regime the angle flow turns off and we are left with

$$
\frac{\partial \vec{V} \cdot \vec{n}}{\rho_{1}+\frac{x_{1}^{2}}{10 \rho_{1}}} \approx \frac{33(2+x)}{2^{\frac{7}{2}} 5}-\frac{9\left(\frac{1}{16}+\frac{2+x}{2^{\frac{3}{2}}}\right)}{10}
$$

(Recall here that $x_{1} \leq \rho_{1}<\epsilon \ll 1$, so that we may make the approximation $\rho_{1}=x_{1}=0$.) This is seen to be positive for all values of $x$ from 0 to 2. Again we see that the flow is inward, since as before $\partial \vec{V}_{\epsilon} \cdot \vec{n}>0$.

3. For $\rho_{1}=\epsilon$, with $\vec{n}=(0,0,0,1,0)=\partial \rho_{1}$,

$$
\begin{aligned}
\frac{\partial \vec{V} \cdot \vec{n}}{\left(\rho_{2}+\frac{x_{2}^{2}}{10 \rho_{2}}\right)}= & \frac{-3\left(2 \rho_{1}+2 \rho_{2}(1-x)\right)\left(\rho_{1}+\frac{x_{1}{ }^{2}}{10 \rho_{1}}\right)\left(x+x_{1}+x_{2}\right)}{4\left(\rho_{1}^{2}+\rho_{2}^{2}+2 \rho_{1} \rho_{2}(1-x)\right)^{\frac{7}{4}}}+ \\
& \left(1-\frac{x_{1}^{2}}{10 \rho_{1}^{2}}\right)\left(\frac{1}{16}+\frac{x+x_{1}+x_{2}}{\left(\rho_{1}^{2}+\rho_{2}^{2}+2 \rho_{1} \rho_{2}(1-x)\right)^{\frac{3}{4}}}\right)
\end{aligned}
$$


The first term is negative and the second positive. Both attain their minimum when $x_{1}$ takes its greatest allowed value of $\rho_{1}$, and for this condition

$$
\begin{aligned}
\frac{\partial \vec{V} \cdot \vec{n}}{\left(\rho_{2}+\frac{x_{2}^{2}}{10 \rho_{2}}\right)}= & -\frac{33 \rho_{1}\left(2 \rho_{1}+2 \rho_{2}(1-x)\right)\left(\rho_{1}+x+x_{2}\right)}{40\left(\rho_{1}{ }^{2}+\rho_{2}{ }^{2}+2 \rho_{1} \rho_{2}(1-x)\right)^{\frac{7}{4}}} \\
& +\frac{9}{10}\left(\frac{1}{16}+\frac{\rho_{1}+x+x_{2}}{\left(\rho_{1}{ }^{2}+\rho_{2}{ }^{2}+2 \rho_{1} \rho_{2}(1-x)\right)^{\frac{3}{4}}}\right) \\
= & \left(\frac{36}{40}-\frac{66}{40}+\frac{6}{40}\left(\frac{\rho_{2}}{\rho_{1}}\right)\left(6 \frac{\rho_{2}}{\rho_{1}}+1-x\right)\right)(>0)+\frac{9}{160}
\end{aligned}
$$

But $\rho_{2}$ is greater than or equal to $\rho_{1}$ with our labeling convention. Using this and setting $x=2$ we find $\partial \vec{V} \cdot \vec{n} /\left(\rho_{2}+\frac{x_{2}^{2}}{10 \rho_{2}}\right) \geq+9 / 160$. Thus the flow without $V_{\epsilon}$ is inward for this part of the boundary. For this part of the boundary $\partial \vec{V}_{\epsilon} \cdot \vec{n} /\left(\rho_{2}+\frac{x_{2}^{2}}{10 \rho_{2}}\right)<0$. However the most negative value it assumes on this boundary is $-\epsilon^{n-4}$, and $n$ can be chosen large enough so that this negative contribution is dominated by the positive $\frac{9}{160}$.

4. Finally for the portion of the boundary defined by $x=\rho_{2}$, with $\vec{n}=\partial x-\partial \rho_{2}$ $=(0,0,1,0,-1)$, the following obtains.

$$
\begin{aligned}
\frac{\partial \vec{V} \cdot \vec{n}}{\rho_{1}+\frac{x_{1}^{2}}{10 \rho_{1}}}= & \frac{a\left(\rho_{2}+\frac{x_{2}^{2}}{10 \rho_{2}}\right)}{\rho_{2}^{2} \rho_{1}^{2}\left(\rho_{2}^{2}+2 \rho_{2} \rho_{1}-2 \rho_{2}^{2} \rho_{1}+\rho_{1}^{2}\right)^{\frac{3}{4}}} \\
& +\frac{3 a \rho_{2} \rho_{1}\left(\rho_{2}+x_{2}+x_{1}\right)}{2\left(\rho_{2}^{2}+2 \rho_{2} \rho_{1}-2 \rho_{2}^{2} \rho_{1}+\rho_{1}^{2}\right)^{\frac{7}{4}}} \\
& +\left(\frac{3\left(\rho_{2}+\rho_{1}-\rho_{2} \rho_{1}\right)\left(\rho_{2}+\frac{x_{2}^{2}}{10 \rho_{2}}\right)\left(\rho_{2}+x_{2}+x_{1}\right)}{2\left(\rho_{2}^{2}+2 \rho_{2} \rho_{1}-2 \rho_{2}^{2} \rho_{1}+\rho_{1}^{2}\right)^{\frac{7}{4}}}\right) \\
& -\left(1-\frac{x_{1}^{2}}{10 \rho_{1}^{2}}\right)\left(\frac{1}{16}+\frac{\rho_{2}+x_{2}+x_{1}}{\left(\rho_{2}^{2}+2 \rho_{2} \rho_{1}-2 \rho_{2}^{2} \rho_{1}+\rho_{1}^{2}\right)^{\frac{3}{4}}}\right)
\end{aligned}
$$

The last term is negative. However it is easy to argue that away from $x=\rho_{2}=$ 2 , where $a=0$, the first term (positive) dominates the last term (negative). 
For $x=\rho_{2}=2$ we have

$$
\begin{aligned}
\frac{\partial \vec{V} \cdot \vec{n}}{F\left(\rho_{1}+\frac{x_{1}^{2}}{10 \rho_{1}}\right)}= & \frac{3\left(2-\rho_{1}\right)\left(2+\frac{x_{2}^{2}}{20}\right)\left(2+x_{1}+x_{2}\right)}{2\left(4-4 \rho_{1}+\rho_{1}^{2}\right)^{\frac{7}{4}}} \\
& -\left(1-\frac{x_{2}^{2}}{40}\right)\left(\frac{1}{16}+\frac{2+x_{1}+x_{2}}{\left(4-4 \rho_{1}+\rho_{2}^{2}\right)^{\frac{3}{4}}}\right)
\end{aligned}
$$

To investigate the positivity of this expression we can let $\rho_{1}$ and $x_{1} \rightarrow 0$, since both are less then $\epsilon$, and obtain

$$
\begin{aligned}
\frac{\partial \vec{V} \cdot \vec{n}}{\rho_{1}+\frac{x_{1}^{2}}{10 \rho_{1}}}= & \frac{3\left(2+x_{2}\right)\left(2+\frac{x_{2}^{2}}{20}\right)}{2^{\frac{7}{2}}} \\
& -\left(1-\frac{x_{2}^{2}}{40}\right)\left(\frac{1}{16}+\frac{2+x_{2}}{2^{\frac{3}{2}}}\right)
\end{aligned}
$$

This has it's minimum value at $x_{2}=0$ and it is positive. And once again $\partial \vec{V}_{\epsilon} \cdot \vec{n}>0$.

We conclude that the flow is inward on all of $\partial N_{\epsilon}$.

\section{Critical Points of $V$}

We now determine the critical points of $V$ and their indexes. Consider the derivatives of $V$ with respect to the three angles:

$$
\begin{aligned}
\frac{\partial V}{\partial \theta_{1}}= & \frac{\left(\rho_{1}+\frac{\left(1-\cos \left(\theta_{1}\right)\right)^{2}}{10 \rho_{1}}\right)\left(\rho_{2}+\frac{\left(1-\cos \left(\theta_{2}\right)\right)^{2}}{10 \rho_{2}}\right) \sin \left(\theta_{1}\right)}{\left(\rho_{1}^{2}+\rho_{2}{ }^{2}+2 \rho_{1} \rho_{2} \cos (\theta)\right)^{\frac{3}{4}}}+ \\
& \frac{\left(1-\cos \left(\theta_{1}\right)\right)\left(\rho_{2}+\frac{\left(1-\cos \left(\theta_{2}\right)\right)^{2}}{10 \rho_{2}}\right)\left(\frac{1}{16}+\frac{3-\cos (\theta)-\cos \left(\theta_{1}\right)-\cos \left(\theta_{2}\right)}{\left(\rho_{1}{ }^{2}+\rho_{2}{ }^{2}+2 \rho_{1} \rho_{2} \cos (\theta)\right)^{\frac{1}{4}}}\right) \sin \left(\theta_{1}\right)}{5 \rho_{1}} \\
\frac{\partial V}{\partial \theta_{2}}= & \frac{\partial V}{\partial \theta_{1}}(1 \leftrightarrow 2)
\end{aligned}
$$




$$
\begin{aligned}
\frac{\partial V}{\partial \theta}= & \left(\rho_{1}+\frac{\left(1-\cos \left(\theta_{1}\right)\right)^{2}}{10 \rho_{1}}\right)\left(\rho_{2}+\frac{\left(1-\cos \left(\theta_{2}\right)\right)^{2}}{10 \rho_{2}}\right) \\
& \left(\frac{\sin (\theta)}{\left(\rho_{1}^{2}+\rho_{2}^{2}+2 \rho_{1} \rho_{2} \cos (\theta)\right)^{\frac{3}{4}}}+\frac{2 \rho_{1} \rho_{2}\left(3-\cos (\theta)-\cos \left(\theta_{1}\right)-\cos \left(\theta_{2}\right)\right) \sin (\theta)}{3\left(\rho_{1}^{2}+\rho_{2}^{2}+2 \rho_{1} \rho_{2} \cos (\theta)\right)^{\frac{7}{4}}}\right)
\end{aligned}
$$

From these expressions it is clear that critical points can occur only for all the $\theta$ 's equal to 0 or $\pi$. To find the critical points of $V$ it thus suffices to find its critical points with respect to $\rho_{1}$ and $\rho_{2}$, assuming fixed $\theta$ 's of 0 or $\pi$. To that end we exhibit contour plots for $V$ with $\Theta=\left(\theta_{1}, \theta_{2}, \theta\right)$ taking these values.

Figure 3 is a contour plot for $V$ in terms of $\rho_{1}$ and $\rho_{2}$ for $\Theta=(\pi, \pi, 0)$. We see that there are three critical points, one at $P_{1,1,0}=\left(\theta_{1}, \theta_{2}, \theta, \rho_{1} \rho_{2}\right) \approx(\pi, \pi, 0,1.5,1.5)$ and a symmetrical pair, one partner of which is at $P_{1,1,0}=\left(\theta_{1}, \theta_{2}, \theta, \rho_{1} \rho_{2}\right) \approx$ $(\pi, \pi, 0,0.7,8.0)$.

Figure 4 is a plot for $V$ for $\Theta=(\pi, \pi, \pi)$. From this plot we see there is a critical point at $P_{1,1,1} \approx(\pi, \pi, \pi, 16,0.6)$.

Similarly Figure 5 is a contour plot for $V$ with $\Theta=(0, \pi, \pi)$. This plot exhibits a critical point at $P_{0,1,1} \approx(0, \pi, \pi, 12,0.6)$.

The plots for the other four possible values of $\Theta$ depict functions with no critical points, and we do not give them here.

From these plots we see that, at these critical points, the eigensigns of the Hessian restricted to the $\rho_{1}-\rho_{2}$-tangent-subspace are positive except for the critical point $P_{1,1,0}=\left(\theta_{1}, \theta_{2}, \theta, \rho_{1} \rho_{2}\right) \approx(\pi, \pi, 0,1.5,1.5)$ of Figure 3 , which is a saddle point with one negative eigensign and one positive one.

From the plots, it seems clear that the addition of the 'small' term $V_{\epsilon}$ (which has no angle dependence) will not introduce any new critical points and will affect only slightly the position of the critical points without changing their indexes. It is also clear from the above expressions for the $\theta$-derivatives of $V$ that the $5 \times 5$ Hessian matrix of $V$ at a critical point has no off-diagonal terms involving $\theta$ 's. The geometries of the three configurations are depicted in Figure 6. 
If one contemplates the types of configuration implied by the values of $\Theta$ at these critical points, it becomes plausible that the signs of the diagonal $\theta$-entries should behave as follows.

1. $P_{1,1,0}$ should have two negative signs for $\theta_{1}$ and $\theta_{2}$, since the particle frames are oppositely aligned to that of the antiparticle (an unstable situation), and one positive sign for $\theta$, since a change of $\theta$ in this case would bring the two particles out of syzygy (a stable situation). Thus the total critical index of $P_{1,1,0} \approx(\pi, \pi, 0,1.5,1.5)$ should be 3 while that of $P_{1,1,0} \approx(\pi, \pi, 0, .7,8)$ should be 2 .

2. $P_{1,1,1}$ should have three negative signs since the frames are anti-aligned and their locations are in "anti-syzygy", and thus a critical index of 3

3. $P_{0,1,1}$ should have two negative signs for $\theta_{2}$ and $\theta$, due to frame misalignment and being out of syzygy, and one positive sign for $\theta_{1}$, due to frame alignment, leading to a critical index of 2

These conclusions are readily confirmed by computing the Hessian of $V$ in the five dimensional space of $\theta_{1}, \theta_{2}, \theta, \rho_{1}, \rho_{2}$ at these critical points.

\section{Critical Indexes of $V$}

Before turning to the case of primary interest, let us consider what the Morse function $V$ tells us when the particles and antiparticle are assumed to move in the Euclidean plane $\mathbb{R}^{2}$, and the frame-space is correspondingly $S O(2)$. In this case each of the critical points lies on a two-dimensional critical submanifold $\mathrm{N}_{i}$ generated by (a) overall rotation $[S O(2)]$ of the points and frames together, and (b) "locked frame rotation" $[S O(2)]$ in which both of the frames rotate clockwise (say) while the antiframe rotates counterclockwise in order that $\theta_{1}$ and $\theta_{2}$ be preserved. The Hessian to be considered is that of the normal subspace to $\mathrm{N}_{i}$. Thus the critical index for $(\pi, \pi, 0,1.5,1.5)$ is 3 , for $(\pi, \pi, 0, .7,8) 2$, for $(\pi, \pi, \pi, 16, .6) 3$ and for $(0, \pi, \pi, 12, .6)$ 
2. By equation (4) we can conclude that (for any choice of the coefficient field $K$ ), $\operatorname{dim} H_{1}$ cannot increase in going from $Q_{1,0}$ to $X_{2,1}=\overline{Q_{2,1}}$ but might decrease by as much as 2 , while $\operatorname{dim} H_{2}$ might change by any integer in the range $[-2,+2]$.

Recall now that $Q_{1,0} \approx \bar{F}$, where $\bar{F}$ is the frame-space of the antiparticle. Since we now know that no new generator of $H_{1}$ can appear in going from $Q_{1,0}$ to $X_{2,1}$, we can conclude that the exchange path in $X_{2,1}$ is either homologous to the cycle in $Q_{1,0}$ corresponding to a $2 \pi$ rotation, or homologous to zero. In Ref. 11 we in fact concluded that the former obtains. (The above conclusion on how $\operatorname{dim} H_{2}$ changes is also consistent with the findings of that reference.)

Now let us turn to the case of primary interest, where the particles and antiparticles are assumed to move in Euclidean 3-space $\mathbb{R}^{3}$. We can anticipate that the critical indexes are considerably enlarged, because both the dimension of the physical Euclidean space and the dimension $d_{F}$ of the frame space have grown. At each of the critical points, the unstable directions amount to rotating the frames out of anti-alignment or the frame-locations out of anti-syzygy keeping the antiparticle frame fixed. The critical submanifolds now are respectively 4 or 5 dimensional ( 3 dimensions for overall rotations, plus $d_{F}$ dimensions for "locked frame rotations" minus 1 dimension over-counted), and the critical indexes refer - as always - to the directions normal to the critical submanifolds.

Consider first the case of $P_{1,1,0}$ : both particle frames anti-aligned but with location-syzygy. There are $2 d_{F}$ unstable directions to rotate the frames of the particles, giving for the critical indexes $\lambda_{1,1,0}^{(1)}=2 d_{F}+1$ for $(\pi, \pi, 0,1.5,1.5)$ and $\lambda_{1,1,0}^{(2)}=2 d_{F}$ for $(\pi, \pi, 0, .7,8)$

In the case of $P_{1,1,1}$, both particle frames are anti-aligned and there is locationanti-syzygy. In addition to the $2 d_{F}$ unstable directions to rotate the frames of the particles there are 2 unstable directions arising from perturbing the locations. (These two directions correspond to the two independent ways that $\vec{\theta}$ can change when the particles can move in three directions. This is in contrast to the two dimensional case for which there is only one independent way.) Thus $\lambda_{1,1,1}=2 d_{F}+2$. 
Finally in the third case of $P_{0,1,1}$ (particles in location-anti-syzygy with the closest particle anti-aligned), there are $d_{F}$ unstable direction to rotate the misaligned frame and, as in the $P_{1,1,1}$ case, there are 2 unstable directions in which to alter the locations, giving $\lambda_{0,1,1}=d_{F}+2$.

Thus for $S^{2}$ frames $\left\{\lambda_{1,1,0}^{(1)}, \lambda_{1,1,0}^{(2)}, \lambda_{1,1,1}, \lambda_{0,1,1}\right\}=\{5,4,6,4\}$ whereas for $S O(3)$ frames $\left\{\lambda_{1,1,0}^{(1)}, \lambda_{1,1,0}^{(2)}, \lambda_{1,1,1}, \lambda_{0,1,1}\right\}=\{7,6,8,5\}$. We see that overall, the minimum index is 4 , which is too large to influence $\operatorname{dim}_{1}(\ldots ; K)$ or $\operatorname{dim}_{2}(\ldots ; K)$ in going from $Q_{1,0}$ to $X_{2,1}$; and we conclude that, for both types of frame,

$$
\operatorname{dim}_{i}\left(X_{2,1} ; K\right)=\operatorname{dim}_{i}\left(Q_{1,0} ; K\right)=\operatorname{dim}_{i}(F ; K), \quad i=1,2 .
$$

It bears emphasis here, that this conclusion has turned out to be independent of the details of the $\rho_{1}-\rho_{2}$ plots given in figures $3-5$. In fact the only type of critical point which did not occur in these plots was one with two negative eigensigns, but such a situation would only have increased the resulting critical indices found above, and therfore would not have disturbed our main conclusion, equation (11). In retrospect we can see that this conclusion follows directly from the observation made earlier, that for our Morse potential, critical points can occur only for all the $\theta$ 's equal to 0 or $\pi$. In particular any critical points in the above plots which might have been overlooked (including ones conceivably introduced by our addition of the $V_{\epsilon}$ term), would have been harmless anyway.

Equation (11) is true for any coefficient-field $K$. We will need it for $K=\mathbb{R}$ (or equivalently $\mathbf{Q}$ ) and $K=\mathbf{Z}_{2}$, the two-element field. Let us take the cases $F=S O(3)$ and $F=S^{2}$ in that order.

1) $F=S O(3)$. We know that $H_{1}\left(S O(3) ; \mathbf{Z}_{2}\right)=\mathbf{Z}_{2}$, the generator being the 1-cycle corresponding to $2 \pi$-rotation of the frame. Thus,

$$
\operatorname{dim} H_{1}\left(X_{2,1} ; \mathbf{Z}_{2}\right)=\operatorname{dim} H_{1}\left(S O(3) ; \mathbf{Z}_{2}\right)=1 .
$$

We can conclude that the rotation remains nontrivial in $X_{2,1}$, and that the exchange one-cycle (which also generates a $Z_{2}$ if it is nontrivial) is either homologous to a frame-rotation, or to zero. By the spin-statistics theorem of Ref. 9 we know 
that the former obtains. Most importantly we conclude that since neither of these cycles is homologous to zero in $X_{2,1}$, this configuration space does in fact "admit spin $1 / 2$ ", as is needed to avoid our entire framework being essentially vacuous as regards questions of spin and statistics. Further since $H_{2}(S O(3) ; \mathbb{R})=0$ and thus

$$
0=\operatorname{dim}_{2}(S O(3) ; \mathbb{R})=\operatorname{dim}_{2}\left(X_{2,1} ; \mathbb{R}\right),
$$

there exists no nontrivial Wess-Zumino term for $X_{2,1}$.

2) $F=S^{2}$. In this case, $H_{1}\left(S^{2} ; Z_{2}\right)=0$, whence

$$
0=\operatorname{dim} H_{1}\left(S^{2} ; Z_{2}\right)=\operatorname{dim} H_{1}\left(X_{2,1} ; Z_{2}\right)
$$

We conclude that in $X_{2,1}$ the exchange one cycle must also be homologous to zero, i.e. that rotation and exchange are both trivial in $H_{1}$. However for $S^{2}$-frames, it is not $H_{1}$ but $H_{2}$ which is responsible for the possibility of half-integer spin ${ }^{7}$. In fact, the $U(1)$ bundles over $S^{2}$ are classified by their "winding number" in $H_{2}\left(S^{2} ; \mathbf{Z}\right)$, and the odd winding numbers belong to spinorial quantum theories. (Such bundles possess a connection whose curvature is an odd multiple of the fundamental "WessZumino term" on $\left.S^{2}\right)$. Now since copies of $\mathbf{Z}$ in $H_{2}(\ldots ; \mathbf{Z})$ show up as copies of $\mathbb{R}$ in $H_{2}(\ldots ; \mathbb{R})$, equation $(11)$ tells us in this case that the 2-cycle corresponding to the " $S^{2}$ of frames" remains nontrivial in $X_{2,1}$;

$$
1=\operatorname{dim}_{2}\left(S^{2} ; \mathbb{R}\right)=\operatorname{dim} H_{2}\left(X_{2,1} ; \mathbb{R}\right)
$$

It follows that the possibility of spinorial states remains as well (which, by the spinstatistics theorem of Ref. 9, augmented in the manner of Ref. 4, is equivalent to the possibility of fermionic statistics). Finally, since $\operatorname{dim} H_{2}\left(X_{2,1} ; \mathbb{R}\right)$ is only 1 , we see that there is no further topologically non-trivial Wess-Zumino term, beyond the one required for nontrivial spin and statistics.

\section{Outlook}

We have shown that for particles moving in $\mathrm{R}^{3}$ and carrying $S O(3)$-frames, the 1cycle of exchange (which is homologous to the 1-cycle of rotating a particle frame) 
is non-trivial in the space $X_{2,1}$. We have also obtained analogous results involving $H_{2}$ for the case of $S^{2}$-frames. It remains to extend these considerations to $X_{3,2}$, and eventually to all the $X_{m, n}=\bar{Q}_{m, n}$, and to their union. One might expect that, in generalizing the Morse potential $V$ to $X_{3,2}$ and beyond, the critical indices would all enlarge considerably because of the greater dimensional configuration space associated with the greater number of particles. Such an enlargement of the critical indexes would imply inductively that $\operatorname{dim}_{1}\left(X_{m, n} ; K\right)$ and $\operatorname{dim}_{2}\left(X_{m, n} ; K\right)$ always remain the same as those of $X_{2,1}$, and hence that the first and second homology groups of $X_{m, n}$ reduce effectively to those of $S^{2}$ or $S O(3)$, as the case may be.

To confirm these expectations via direct generalization of our potential $V$ to arbitrary $X_{m, n}$ would seem difficult, because our analysis of the critical points in Section 7 was graphical and not analytic. Instead of this, one could try to construct the flow $\xi^{a}$ directly by extending to arbitrary configurations the more easily defined flow à la Ref. 11 which retracts a neighborhood of $X_{m+1, n+1}$ back to $X_{m, n}$. In effect, this is what we have done above for $(m, n)=(1,0)$, and a scheme for doing something similar in general does not seem too difficult to devise.

An alternative approach would be to try to generalize the Mayer-Vietoris techniques of Ref. 11, by means of which results analogous to those of the present paper were found for $X_{2,1}$ in the rather simpler, two-dimensional situation. Such techniques might ultimately furnish more information on the homology of configuration space, but in our experience, they have been more cumbersome in application than the Morse-theory techniques of the present paper, and their complexity escalates as the spatial dimension grows.

We conclude by returning briefly to the 2-dimensional situation with which we began Section 8, which concerns particles moving in $\mathbb{R}^{2}$ and carrying $S O(2)$ frames. In that situation, we showed above that, although $H_{1}$ can only decrease in going from $X_{1,0}$ to $X_{2,1}, H_{2}$ can (and in fact does) increase, giving rise to the possibility of a Wess-Zumino term in the Action. In this connection, an interesting question would arise if the critical indices were indeed to enlarge considerably when going to 
$X_{3,2}$ and beyond, as suggested above in the 3-dimensional case; for this would mean that $H_{2}$ would necessarily remain nonzero, and the Wess-Zumino possibility would persist. We have shown elsewhere ${ }^{11}$ that $H_{2}\left(X_{2,1} ; Z\right)=Z$ and have exhibited a nontrivial closed two form defined on $Q_{2,1}$ which vanishes at the lower stratum $Q_{1,0}$. The question arises as to what is expected about realizing, say, $H^{2}\left(X_{3,2}\right)$ by closed two forms. Should one expect a closed form on $Q_{3,2}$ which reduces on approach to $Q_{2,1}$ to the form of Ref. 11? We have sought such a form to no avail. Does this mean that $H_{2}\left(X_{3,2}\right)=0$ ? Or does it mean that $H_{2}\left(X_{3,2}\right)$ remains non-zero, but it cannot be realized by closed two forms which go over continuously from $Q_{3,2}$ to $X_{2,1}$, and if so, then what is the physical significance of this impossibility? The answers await further analysis.

\section{Acknowledgments}

We would like to thank M. Rothenberg for suggesting the use of Morse Theory and explaining how it could be applied to our configuration spaces. We are also grateful to A.P. Balachandran for extensive discussions during the initiation of this work. This work was supported by a National Science Foundation cooperative research grant, INT 8814944. In addition, R.D.S. was partially supported by the National Science Foundation under grant number PHY 9307570 and by research funds from Syracuse University and S.S by FORBAIRT. W.D.M. would like to thank the Dublin Institute for Advanced Studies for the hospitality shown during several visits when much of this work was done, and R.D.S would like to thank Trinity College Dublin and the Dublin Institute for Advanced Studies for hospitality during a series of visits.

\section{References}

[1] D. Finkelstein and J. Rubinstein, J. Math. Phys. 9, 1762(1968)

[2] Cf. R. F. Streater and A. S. Wightman, PCT, Spin and Statistics, and All That (Benjamin, New York, 1964); U. H. Neider and L. O'Raifeartaigh, Fortschr. 
Phys. 22, 111, 131 (1974); and references therein.

[3] J. L. Friedman and R. D. Sorkin, Commun. Math. Phys.86, 483, 501 (1983) .

[4] R. D. Sorkin, Commun. Math. Phys.115, 421 (1988). See also Ref. 3 and A. P. Balachandran, G. Marmo, B. S. Skagerstam and A. Stern, Classical topology and Quantum States (World Scientific, 1991) for further references.

[5] R. D. Tscheuschner, Int. J. Theor. Phys.28, 1269 (1989)

[6] D. J. Simms and N. M. J. Woodhouse: Lectures on Geometric Quantization: Lecture Notes in Physics, Vol. 35 , (Springer, 1977) Appendix A.

[7] R. D. Sorkin Phys Rev. D27, 1787 (1983)

[8] A. P. Balachandran, A. Daughton, A.-C Gu, G. Marmo, R. D. Sorkin and A. M. Srivastava, Mod. Phys. Lett. A5, 1575 (1990)

[9] A. P. Balachandran, A. Daughton, A.-C Gu, G. Marmo, R. D. Sorkin and A. M. Srivastava, Int. J. Mod. Phys. A 8, 2993 (1992)

[10] M. Bourdeau and R. D. Sorkin,Phys. Rev. D45 , 687 (1992)

[11] A. P. Balachandran, W. D. McGlinn, L. O'Raifeartaigh, S. Sen and R.D. Sorkin, Int. J. Mod. Phys. A 7, 6887 (1992)

[12] See for example B.A. Dubrovin, A.T. Fomenko and S.P. Novikov Modern Geometry - Methods and Applications, Part III (Springer - Verlag, 1990); R. Bott, "Lectures on Morse Theory, Old and New", Bull. Am. Math. Soc 7(\# 2):331$358(1982)$. 


\section{Figure Captions}

- Fig.1 Depiction of subspaces $\Sigma, \Sigma_{t}$, and $M_{t}$ and the retraction flow through $x$ . See text.

- Fig.2. Depiction of subspaces involved when retracting through a critical point. See text .

- Fig.3 . Contour plot for $V$ for $\Theta=(\pi, \pi, 0)$, showing three critical points, one at $\left(\theta_{1}, \theta_{2}, \theta, \rho_{1} \rho_{2}\right) \approx(\pi, \pi, 0,1.5,1.5)$ and a symmetrical pair, one partner of which is at $\left(\theta_{1}, \theta_{2}, \theta, \rho_{1} \rho_{2}\right) \approx(\pi, \pi, 0,0.7,8.0)$.

- Fig.4. Contour plot for $V$ for $\Theta=(\pi, \pi, \pi)$ showing a critical point at $\left(\theta_{1}, \theta_{2}, \theta, \rho_{1} \rho_{2}\right) \approx(\pi, \pi, \pi, 16,0.6)$.

- Fig.5. Contour plot for $V$ with $\Theta=(0, \pi, \pi)$, showing a critical point at $\left(\theta_{1}, \theta_{2}, \theta, \rho_{1} \rho_{2}\right) \approx(0, \pi, \pi, 12,0.6)$.

- Fig.6. Geometries of the three configurations which exhibit critical points. The heavy arrow represents the antiparticle and the light arrows the particles. 